\title{
Aprendizaje del mapunzugun desde metodologías propias: perspectivas y avances para la revitalización de lenguas originarias ${ }^{1}$
}

\author{
Mapunzungun learning from Mapunche methodologies: perspectives \\ and advances for the revitalization of native languages
}

Aprendizagem do Mapunzugun a partir de metodologias próprias:
perspectivas e avanços para a revitalização de línguas originárias

Kimgeken kewüh mapun az kimün mew: Fij amulgen ka güneltun mew ñi newenyegetuael fijke az mapun kewüh

\author{
Gabriel Llanquinao Llanquinao ${ }^{2}$ \\ Universidad Católica de Temuco, Chile \\ Gastón Salamanca Gutiérrez ${ }^{3}$ \\ Universidad de Concepción, Chile \\ Fernando Teillier Coronado ${ }^{4}$ \\ Universidad de Las Américas, Chile
}

Recepción: 15/03/2019

Evaluación: 15/05/2019

Aceptación: 23/05/2019

Artículo de Investigación - Revisión

DOI: https://doi.org/10.19053/01227238.9918

$1 \quad$ Este artículo es parte de la tesis doctoral "Aprendizaje del mapunzugun en la visión de los hablantes nativos: bases lingüísticas para su revitalización", investigación en curso del académico Gabriel Llanquinao para optar al grado de doctor en Lingüística por la Universidad de Concepción. Utilizamos en este texto los conceptos "mapunzugun" y "mapunche" (en vez de "mapuzugun" y "mapuche"), pues aquellos dan cuenta de manera más exhaustiva de "lo propio", por ejemplo, en el ámbito lingüístico. En estricto rigor, "mapuzugun" da cuenta de una verbalización vinculada con conflictos de tierra, lo que históricamente se confundió con el nombre de la lengua. Asimismo, estos conceptos sintonizan plenamente con los focos que se desplegarán y relevarán en nuestra presentación, toda vez que el énfasis está puesto, precisamente, en la perspectiva de los propios hablantes nativos como fundamento basal para la revitalización de la lengua. Eso sí, aunque por las razones expuestas en nuestra presentación utilizamos sistemática y consistentemente "mapunzugun" y "mapunche", en las citas textuales y en las referencias bibliográficas mantenemos los conceptos utilizados por los autores respectivos, donde, por cierto, por lejos es mayoritario el uso de "mapuche" y "mapuzugun/mapudugun".

2 Doctorando del Programa de doctorado en Lingüística de la Universidad de Concepción, Chile. Docente de la Universidad Católica de Temuco. Correo electrónico: gllanqui@uct.cl.

3 Doctor en Lingüística. Docente de pre y postgrado de la Universidad de Concepción, Chile. Correo Electrónico: gsalaman@udec.cl.

4 Doctor en Lingüística. Docente de la Universidad de Las Américas, Chile. fernandoteillier@gmail.com. 


\section{RESUMEN}

En este artículo se revisan los aportes de algunos estudios en relación con la enseñanza-aprendizaje de lenguas originarias, especialmente del mapunzugun. Considera para ello diversas perspectivas, aunque se focaliza en aquellas que emergen desde el propio patrimonio lingüístico. Así, se presentan las propuestas de metodologías de enseñanza dentro de un contexto de revitalización, su relación con la lingüística aplicada y la articulación de esta última con perspectivas de enseñanza de la lengua basada en las epistemologías y metodologías de la lengua vernácula. Junto con ello, se presentan algunas consideraciones críticas respecto del lugar que ocupa el mapunzugun en el contexto del Programa de Educación Intercultural Bi- lingüe en Chile y una aproximación a los conceptos vernáculos de kimün y rakizuam como articuladores claves de cualquier metodología de enseñanza-aprendizaje basada en perspectivas propias. Una conclusión prominente de esta presentación es que es necesario el desarrollo y aplicación de metodologías de enseñanza de la lengua que provengan de la práctica sociocultural de la lengua a revitalizar, relegando a un segundo plano las lógicas teórico/pedagógicas provenientes de la lengua dominante.

Palabras clave: Revista historia de la educación latinoamericana; etnolingüística; enseñanza-aprendizaje del mapunzugun; metodologías propias; revitalización.
In this article the contributions of some studies in relation to the teaching-learning of native languages, especially from the Mapunzugun, are reviewed. For this purpose, different perspectives are considered, although the focus is on those that emerge from the linguistic heritage of the language studied. Thus, the proposals of teaching methodologies within a context of revitalization, their relationship with applied linguistics and the articulation of the latter with perspectives of language teaching based on the epistemologies and methodologies of the vernacular language are presented. Along with this, some critical considerations regarding the place occupied by Mapunzugun in the context of Intercultural Bilingual Education in Chile and an approach to the vernacular concepts of kimün and rakizuam as key articulators of any teaching-learning methodology based on Mapunche perspectives are presented. A prominent conclusion of this work is that it is necessary to develop and apply language teaching methodologies that come from the socio-cultural practice of the language to be revitalized, relegating the theoretical/pedagogical logics coming from the dominant language to a second level.

Keywords: Journal History of Latin American Education; ethnolinguistics; mapunzugun teaching-learning; Mapunche methodologies; revitalization.

\section{RESUMO}

Neste artigo são revisados os aportes de alguns estudos sobre ensino-aprendizagem de línguas originárias, especialmente do Mapunzugun. Para tanto, consideram-se diversas perspectivas, ainda que o enfoque esteja naquelas que emergem do próprio patrimônio linguístico. Assim, apresentam-se propostas de metodologias de ensino dentro de um contexto de revitalização, sua relação com a linguística aplicada e a articulação desta com perspectivas de ensino de língua baseadas em epistemologias e metodologias da língua vernácula. Ademais, apresentam-se algumas considerações críticas sobre o lugar ocupado pelo Mapunzugun no contexto da Educação Intercultural Bilíngue no Chile e uma aproximação aos 
conceitos vernáculos de kimün y rakizuam como articuladores-chave de qualquer metodologia de ensino-aprendizagem fundada em perspectivas próprias. Uma conclusão relevante deste trabalho indica a necessidade de desenvolvimento e aplicação de metodologias de ensino de língua que provenham da prática sociocultural da língua a revitalizar, relegando a um segundo plano as lógicas teórico/pedagógicas oriundas da língua dominante.

Palavras-chave: Etnolinguística; ensino-aprendizagem do Mapunzugun; metodologias; próprias; revitalização.

\section{PIKÜNOGETUN}

Tüfa chi zugu mew güneltugey chem ñi azkonkülen mufü pin mew chew ñi wepümtugen kimeltugen ta kewüh wej kake kishu azmapu kewüh, feygen ta mapunzugun. Femechi mew tukugey fij az güneltun ñi mülewmen mew wej zoygey kishu az güneluwün kimün yenieyewmelu ta elürpuel chi kewüh. Fey wüxampüramgey fij azküno kimeltugen ta kewüh chew ñi newenyegetun, fij günel azküno kewüh kimeltun kimün ka tüfeychi kishu azkünow mapun kimüngewmechi kewüh kimün mew. Ka xürüwüngechi zugu mew, wepümtugey fij zuguntukun güneltun, chumgechi ñi yeniegen mapunzugun fey ti chi epu rume kimeltuwün-kewüh zugu piel mew, ka may chum nüwmen mapun az kimün ka rakizuam mew feygen ñi pelom püramgeal mapun kimeltuwün zugu kizugenkülen chi azkünowün kimeltun. Kiñe yaf püram pefal zugu tüfachi pin mew rume fanentugen ñi amuael wej ñi konael tüfachi mapun azküno kimeltuwün nüwmeyelu ta mapun azmogen mew, femechi ñi newenyegetuael mapun kewüh, femechi ñi inakonkülepuael wigka kimün güneltun kimeltun wenunyeniel chi kewüh mew.

Zichul zugun: Revista Historia de la Educación Latinoamericana; etnolingüistica; Mapun kimeltuwün kewüh; kishu azkünow kimün; newenyegetun.

\section{INTRODUCCIÓN}

Desde los primeros contactos con la sociedad occidental, las sociedades indígenas, en particular la mapunche, han sido estudiadas desde distintos ámbitos del conocimiento. Uno de ellos se refiere al patrimonio lingüístico, a partir de los llamados cronistas y misioneros, en cuyos estudios es posible identificar sus propósitos como esencialmente orientados hacia la evangelización y alfabetización. En efecto, en un sentido amplio, los estudios y descripciones de la lengua, en este caso del mapunzugun, provenientes de las fuentes antes señaladas, constituyen parte relevante de la documentación lingüística, así como también sus descripciones y procesos de documentación han sido utilizados con frecuencia para profundizar acerca de la lengua en términos académicos, es decir, en el estudio de la lengua como un sistema. A partir de estos trabajos pioneros, se ha desarrollado una tradición de estudios e investigaciones acerca del mapunzugun centrada fundamentalmente en aspectos descriptivos y de documentación 
Así, se han realizado exhaustivas investigaciones sobre aspectos fonético-fonológicos ${ }^{5}$ y gramaticales ${ }^{6}$.

Por otra parte, en los últimos años se han desarrollado investigaciones en el ámbito de la sociolingüística, sobre todo relacionadas con la descripción del estado de la lengua en términos del número y actitudes de sus hablantes ${ }^{7}$, y la revitalización lingüística ${ }^{8}$. A su vez, es posible encontrar estudios y publicaciones que abordan el aprendizaje y enseñanza del mapunzugun para contextos formales e informales ${ }^{9}$, si bien en menor medida.

El presente trabajo se sitúa en estos últimos contextos (revitalización lingüística y enseñanza del mapunzugun), toda vez que se aproxima a nociones propias de la lengua y su relación con los procesos de enseñanza-aprendizaje que ella misma contiene y despliega. En este sentido, se propone un acercamiento a la enseñanza del mapunzugun que asuma, en un diálogo permanente, una relación productiva entre la lingüística descriptiva, la revitalización lingüística y la lingüística aplicada, desde las epistemologías y metodologías propias de los hablantes. Este artículo busca, en consecuencia, relacionar los conceptos acerca de las propuestas de aprendizaje y enseñanza de lenguas, fundamentalmente de lenguas originarias, a fin de generar propuestas de enseñanza para distintos contextos, tanto formales como informales. En el ámbito formal, nos referimos en esencia al contexto escolar; en particular al Programa de Educación Intercultural Bilingüe. En el segundo contexto, hacemos referencia, por ejemplo, a los ámbitos familiar y comunitario.

En definitiva, nos ocupamos del desarrollo de una perspectiva teórica y metodológica que permita abordar el estudio del mapunzugun de una manera apropiada, de modo que se posibilite sustentar propuestas que orienten proce-

5 Entre otros: Gastón Salamanca Gutiérrez y Elizabeth Quintrileo Llancao, "El mapuche hablado en Tirúa: fonemas segmentales, fonotaxis y comparación con otras variedades", Revista de Lingüística teórica y aplicada, n.o 47 (2009): 13-35; Scott Sadowsky et al., "Mapudungun", Journal of the International Phonetic Association, n.o 43 (2013): 87-96; Makarena Sánchez Pérez y Gastón Salamanca Gutiérrez, "El mapuche hablado en Lonquimay: Fonemas segmentales, fonotaxis y comparación con otras variedades", Literatura y Lingüística, n.o 31 (2015): 295-332.

6 Entre otros: Arturo Hernández Sallés, Nelly Ramos Pizarro y Rosa Huenchulaf, Gramática Básica de la Lengua Mapuche (Temuco: Universidad Católica de Temuco-Conadi, 2006). Adalberto Salas Santana, El mapuche o araucano. Fonología, gramática y antología de cuentos (Santiago: Centro de Estudios Públicos, 2006); Fernando Zúñiga, Mapudungun. El habla mapuche (Santiago de Chile: Centro de Estudios Públicos, 2006); Rodrigo Becerra Parra, "Una propuesta funcional y tipológica de descriptores sintácticos para el mapudungun", Alpha, n.o 32 (2011): 111-125; Rodrigo Becerra Parra, "Expresión de la causalidad en la lengua mapuche" (tesis de maestría en Lingüística aplicada, Universidad de Concepción, Chile, 2011).

7 Entre otros: Fernando Zúñiga, “Mapudunguwelaymi am? ‘¿Acaso ya no hablas mapudungun?’. Acerca del estado actual de la lengua mapuche”, Estudios Públicos, n.o 105 (2007): 9-24; Hans Gundermann Kröll et al., Perfil sociolingüístico de comunidades mapuche de la VIII, IX y X Región. Informe de Resultados 2008 (Santiago de Chile: Universidad Tecnológica Metropolitana-Conadi, 2008); Hans Gundermann Kröll et al., "Permanencia y desplazamiento, hipótesis acerca de la vitalidad del mapuzungun", Revista de Lingüística Teórica y Aplicada, n.o 47, fasc. 1 (2009): 37-60; Fernando Wittig González, "Desplazamiento y vigencia del mapudungún en Chile: un análisis desde el discurso reflexivo de los hablantes urbanos", Revista de Lingüística Teórica y Aplicada, n.o 47, fasc. 2 (2009): 135-155.

8 Entre otros: María Catrileo Chiguailaf, "Revitalización de la lengua mapuche en Chile”, Documentos Lingüísticos y Literarios, n.o 28 (2005): 10-17; Héctor Mariano Mariano et al., “¿Mongeley kam mongelelay chi mapudungun waria mew? Vitalidad y representación social del mapudungún en Santiago de Chile", Romanitas, vol. 4, n.o 2 (2010): 42-69; Cristian Lagos Fernández. "El mapudungún en Santiago de Chile: Vitalidad y representaciones sociales en los mapuches urbanos", Revista de Lingüística Teórica y Aplicada, n.o 50, fasc. 1 (2012): 161-190; Fernando Teillier Coronado, "Vitalidad lingüística del mapudungun en Chile y epistemología del hablante", Revista de Lingüística Teórica y Aplicada, n.o 51, fasc. 1 (2013): 53-70.

9 Entre otros: Aldo Olate Vinet y Marisol Henríquez Barahona, "Actitudes lingüísticas de profesores mapuche de Educación Básica: vigencia y enseñanza del mapudungun en el contexto educativo", Literatura y Lingüística, n.o 22 (2010): 103-116. 
sos de revitalización desde los contextos y actores implicados. Se trata de pensar y repensar la revitalización de lenguas minorizadas y amenazadas, en este caso el mapunzugun o lengua mapunche, a partir de una articulación funcional entre la etnolingüística, la lingüística aplicada y la lingüística descriptiva (en particular, la documentación lingüística).

\section{Mapunzugun y documentación lingüística}

Un primer foco en esta tarea consiste en asumir como punto de partida la caracterización y descripción del mapunzugun desde los primeros estudios, es decir, considerar el abordaje de la lengua desde los trabajos propios de la documentación lingüística anterior y contemporánea a la conquista de la Araucanía (desde los siglos XVII, hasta principios del XX). Esto permite recoger una serie de conceptos que constituyen las bases necesarias para, por un lado, profundizar en los alcances de significado y sentido que la lengua se ha otorgado y, por otro, relacionar esos ámbitos desde su asimilación socio y etnolingüística. Se trata, en definitiva, de recuperar la conformación epistemológica de las propuestas de comprensión de la lengua desde su primera documentación, a fin de relevar la recuperación escrita de las primeras lecturas a la lengua proveniente de hablantes nativos y, también, de actualizar dichos conceptos y nociones sobre los rasgos de la lengua desde una perspectiva ubicada fundamentalmente dentro de un orden socio y etnolingüístico.

Si bien dichos estudios acerca del mapunzugun corresponden a los propósitos propios de la época, nos acercan a la posibilidad de relevar conceptos y orientaciones que permitan configurar aspectos teóricos y metodológicos sobre el aprendizaje de la lengua. Desde luego, será importante tener presente no solo los rasgos lingüísticos y etnolingüísticos respecto del estado de la lengua propuestos en dichos estudios, sino, también, potenciar su uso como recursos que la lengua pueda desplegar junto con sus hablantes, en el contexto de relaciones interétnicas $\mathrm{y}$, por lo mismo, interculturales.

\section{Situación sociolingüística y enseñanza del mapunzugun}

El panorama actual, asociado a la progresiva y notoria pérdida del mapunzugun, en especial entre las nuevas generaciones y en distintos contextos territoriales ${ }^{10}$, permite visualizar escenarios y experiencias de enseñanza desde distintas vertientes o actores e instituciones, las que se destacan - entre otros aspectos - por favorecer procesos de posicionamiento identitario, principalmente en las nuevas generaciones. En efecto, estas observan como aspecto relevante la reafirmación de la identidad, sobre todo visibilizada a través de la práctica del mapunzugun en el medio urbano. Así, pareciera promisorio pensar que estas experiencias abren posibilidades de abordar de manera cada vez más apropiada

10 Hans Gundermann Kröll et al., Perfil sociolingüístico; Hans Gundermann Kröll et al., “Permanencia y desplazamiento”, 37-60. 
la enseñanza-aprendizaje de la lengua originaria y, en consecuencia, posibilitar transmisiones más cercanas y coherentes con el modelo del patrimonio lingüístico del pueblo mapunche. Un rasgo prominente de estas experiencias es que se centran en los actores que la promueven, lo cual, sin duda, es un valor; sin embargo, al implementarse muchas veces desde la intuición, generan escenarios inciertos ${ }^{11}$.

Asimismo, la mayoría, si es que no todas, las experiencias de enseñanza del mapunzugun promueven el uso de la escritura como centro, lo cual constituye, a lo menos, un distanciamiento respecto de la naturaleza de tradición oral de la lengua. Por otra parte, las experiencias de enseñanza son expresadas en términos fundamentalmente formales (escuelas y talleres), lo que se distancia de la lógica de reproducción de la lengua, evidenciando una brecha entre el conocimiento lingüístico mapunche y el académico, toda vez que la transmisión, y por lo tanto el mantenimiento del mapunzugun, tiene como eje la transmisión intergeneracional dentro de una oralidad situada siempre en contextos de uso. En este sentido, desde nuestra perspectiva, y desde la revitalización lingüística, se propone el desarrollo de un proceso de enseñanza de la lengua fundamentado en las bases culturales que la lengua contiene y promueve, lo que permite comprender la relación y expresión entre lengua y pensamiento, y no solamente nociones basadas en la comprensión de la lengua como sistema lingüístico a describir y documentar.

El reconocimiento de prácticas de enseñanza-aprendizaje del mapunzugun basadas en las propias formas de transmisión de la lengua se vincula con un posicionamiento identitario, social y cultural que reivindica aspectos culturales y lingüísticos como expresión o respuesta a prácticas de discriminación. En sentido estricto, se trata del empoderamiento de la lengua minorizada como eje de las diferencias con el otro, favoreciendo la emergencia del patrimonio cultural y lingüístico que la lengua contiene como expresión de identidad.

En este contexto, resulta relevante incorporar a la discusión las perspectivas de los propios hablantes en el contexto familiar y comunitario, para recoger aportes relacionados con la enseñanza de la lengua dentro de un proceso propio de aprendizaje. Por otra parte, a partir de la observación de procesos de mantenimiento del mapunzugun en contextos familiares y comunitarios, es posible relevar un conjunto de ejes que permiten visualizar modos y prácticas de aprendizaje de la lengua, generando patrones susceptibles de ser replicados tanto en el ámbito de la comunidad, como en el de instituciones tales como la escuela. Se trata, entonces, de relacionar los saberes epistemológicos y metodológicos tradicionales de transmisión del mapuzungun (a partir de la intergeneracionalidad y la oralidad) con las ofertas formales (en específico, las provenientes de la escuela), a fin de crear una metodología inclusiva y en permanente diálogo, pero que considere como eje fundamental del despliegue de los procesos de enseñanza las expectativas y necesidades de la comunidad de hablantes. Para efectos del

11 María Catrileo Chiguailaf, La lengua mapuche en el siglo XXI (Valdivia: Universal Austral de Chile, 2010). 
presente artículo, nos centraremos básicamente en la articulación del Programa de Educación Intercultural Bilingüe (PEIB) con y desde las epistemologías y metodologías de enseñanza del mapunzugun provenientes de la comunidad de hablantes de la lengua.

\section{Revitalización lingüística, metodologías propias y lingüística aplicada}

Un elemento central dentro de los derechos socioculturales de un pueblo lo constituye el mantenimiento y vitalización de su lengua, lo que ha sido recogido tanto por los hablantes como por las instituciones del Estado; sin embargo, estas últimas no han propuesto un cuerpo coherente y explícito de políticas de planificación lingüística, configurando "un área que, tanto a nivel político como académico, aún no ha sido desarrollada en propiedad en el país, alejándonos de otras realidades (por ejemplo, Canadá, EE. UU., México, Bolivia)"12. Pese a esto, y debido a consideraciones de tipo políticas tendientes a aminorar las tensiones sociales dentro de estados pluriétnicos, de modo tal de generar climas sociales estables que garanticen la llegada de inversores externos dentro de las reglas del juego establecidas por instituciones multilaterales como el Banco Mundial o el Fondo Monetario Internacional, se consideró la educación como un eje central de la revitalización de las culturas indígenas, esto como parte del "nuevo trato" que el Estado quería establecer con estas poblaciones ${ }^{13}$. En este contexto, se desarrollaron diversas iniciativas, tales como la generación "de un grafemario para la lengua mapuche o, más recientemente, el Programa de Educación Intercultural Bilingüe (PEIB) y la promoción de talleres urbanos de lenguas indígenas desde CONADI"14.

De estas iniciativas, la más relevante, tanto en términos de recursos asignados como de territorio y población considerada, es el Programa de Educación Intercultural Bilingüe, el cual desarrolla las siguientes estrategias: implementación del sector/asignatura de Lengua Indígena, revitalización cultural y lingüística, interculturalidad en el espacio escolar y estrategias de bilingüismo. Sin embargo, de acuerdo con investigaciones recientes, la aplicación del PEIB no logra responder a sus propios lineamientos estratégicos, ya que: (1) no es una herramienta de revitalización de las lenguas indígenas, por lo que sus carencias conceptuales y metodológicas son evidentes, (2) no cumple con su promesa de ser bilingüe (sobre todo en el ámbito urbano) y (3) no es intercultural, pues no hay un diálogo simétrico entre las dos culturas en contacto: el indígena se ve obligado a aprender el español y todo el entramado simbólico de la sociedad chilena (contenidos que no son optativos), lo que redunda en que los contenidos indígenas no parecen significativos, ni en el currículo manifiesto ni en el ocul-

12 Cristián Lagos Fernández y Marco Espinoza Alvarado, "La planificación lingüística de la lengua mapuche en Chile a través de la historia", Lenguas Modernas, n.o 42 (2013): 48.

13 Cristián Lagos Fernández, "El Programa de Educación Intercultural Bilingüe y sus resultados: ¿perpetuando la discriminación?”, Pensamiento Educativo. Revista de Investigación Educacional Latinoamericana, vol. 52, n.o 1 (2015): 84-94.

14 Lagos y Espinoza, "La planificación lingüística”, 48. 
to $^{15}$. Esto no debe extrañar, ya que el PEIB no considera elementos propios de la sociolingüística y la antropología lingüística dentro de sus estructuras teórico/ metodológicas, las que podrían implicar un fuerte aporte al desarrollo de perspectivas de planificación y revitalización lingüísticas.

En este contexto, un aspecto relevante consiste en el relevamiento del hablante y de las comunidades de habla como elementos centrales dentro de la realización de procesos de planificación y revitalización. En palabras de Catrileo: "Si una lengua está perdiendo vitalidad, debido al escaso interés que muestra la comunidad, es preciso introducir programas de intervención para cambiar las actitudes y reforzar la utilización de la lengua"16; es decir, es preciso situar el proceso de (re)vitalización lingüística desde la participación activa y comprometida de la comunidad de hablantes, tanto en términos del reconocimiento de las características internas de uso de la lengua, como de las relaciones socioculturales extracomunitarias. Así, como posible salida a esta dinámica, más que hacer hincapié en las deficiencias del PEIB, el desafío consiste en acompañar las iniciativas de planificación que están en desarrollo, tales como los talleres de lengua mapuche impartidos en las ciudades y el Programa de Educación Intercultural Bilingüe ${ }^{17}$, a partir del desarrollo de perspectivas de revitalización basadas en las epistemologías y metodologías de enseñanza de la lengua propias de la comunidad de hablantes.

En consonancia con lo anterior, la presente propuesta pretende, desde el diálogo entre el PEIB y la comunidad de hablantes, proponer y aplicar una metodología de revitalización del mapunzugun que engarce al PEIB con las comunidades, potenciando la emergencia de propuestas y aplicaciones de (re)vitalización que emerjan desde los mismos hablantes. Es a partir de la generación de políticas lingüísticas y socioculturales que tengan arraigo en la comunidad, y que se centren en el establecimiento de un diálogo de (re)creación intergeneracional y comunitaria del uso del mapunzugun, que se debe considerar la inclusión dialogada (comunidad / Estado) del PEIB. Esto puede ser un factor gravitante a la hora de generar sinergias que favorezcan los procesos comunitarios de (re) vitalización lingüística, a la vez que posibilitaría el potenciar las estrategias del PEIB.

\section{Revitalización lingüística}

Desde la literatura, Hinton y Hale emplean el término "revitalización lingüística" en un sentido amplio ${ }^{18}$. En el sentido más extremo, se refiere al desarrollo de procesos que resulten en el restablecimiento de una lengua que ha cesado de

15 Lagos, "El Programa de Educación".

16 Catrileo, "Revitalización de la lengua mapuche", 12.

17 Lagos y Espinoza, "La planificación lingüística”, 47-66.

18 Leanne Hinton y Ken Hale, eds., The Green Book of Language Revitalization in Practice (San Diego, CA: Academic Press, 2001). 
ser la lengua con la cual se comunica una comunidad de habla y la vuelta a un uso total de esta en todos los aspectos de la vida.

En un sentido no tan extremo, la revitalización puede darse en una situación de pérdida de uso de una lengua, en la que esta es la primera lengua de muchos niños y es usada en muchos hogares de manera comunicativa, pero se encuentra perdiendo terreno frente a otras (el caso del Irlandés y el Navajo). En estas comunidades de habla, la revitalización debería significar un viraje en el declive del uso de la lengua. Por su parte, King define la revitalización lingüística como el intento de añadir nuevas formas y funciones a una lengua que se encuentra amenazada de desplazamiento o muerte con el propósito de incrementar su uso y usuarios $^{19}$.

Dentro de las ofertas de revitalización lingüística, es posible reconocer al menos tres propuestas: el modelo de Reversión del Desplazamiento de Lenguas o Reversing Language Shift ${ }^{20}$, el modelo de Ruedas de Fuego ${ }^{21}$ y el Modelo de Perfil Social $^{22}$; sin embargo, estos modelos de revitalización se establecen a partir de una relación operativa, basada en perspectivas estáticas, es decir, no sujeta a lecturas inmediatas de retroalimentación del proceso de revitalización lingüística, sino, más bien, temporalizadas sincrónicamente desde una construcción y análisis de datos que no considera la participación activa de la comunidad de hablantes. Además, las propuestas mencionadas reconocen como indicadores de vitalidad la inserción del hablante en las estructuras socioeconómicas y culturales dominantes, con todo lo que ello implica.

Esto es visible, en el caso de Strubell, en los campos de uso de la lengua propuestos: oferta/demanda y mercado laboral. Por su parte, la propuesta de Fishman (Graded Intergenerational Dislocation Scale) si bien establece como centro de la vitalidad de una lengua la transmisión intergeneracional, releva como indicadores de un alto grado de vitalidad la existencia de contextos de uso institucionales propios de la cultura dominante y la presencia activa de la lengua en medios de comunicación, lo que se encuentra reñido con los contextos de uso tradicionales y el ejercicio activo de la oralidad dentro de la producción y reproducción de la lengua. El Modelo de Perfil Social, en tanto, si bien considera la incorporación activa de los hablantes en el desarrollo de propuestas de revitalización lingüística, dicha participación se encuentra subordinada a propuestas de perfil altamente academicista. Así, entonces, las propuestas de revitalización presentadas fijan la vitalidad del uso de la lengua en un entorno único, capitalista y occidental $^{23}$, por lo que su pertinencia resulta altamente cuestionable dentro

19 Kendall King, Language Revitalization Processes and Prospects (Clevedon: Multilingual Matters, 2001).

20 Joshua A. Fishman, Reversing Language Shift: Theoretical and Empirical Foundations of Assistance to Threatened Languages (Clevedon: Multilingual Matters, 1991).

21 Miquel Strubell, “Can Sociolinguistic Change be Planned?", en Proceedings of the 1st European Conference, "Private Foreign Language Education in Europe. Its contribution to the Multilingual and Multicultural Aspect of the European Union" (Thessaloniki: Palso, 1998), 23-31.

22 Jeroen Darquennes, "Paths to Language Revitalization", en Contact Linguistics and Language Minorities, ed. de Jeroen Darquennes, vol. XXX, Plurilingua (St. Augustin: Asgard, 2007), 61-76.

23 Fernando Teillier Coronado, “Configuración de realidad y lengua: bases de una epistemología del mapunzugun” (tesis de doctorado en Lingüística, Universidad de Concepción, Chile, 2015). 
de un contexto de revitalización de lenguas originarias; en este caso particular, del mapunzugun.

Se trata, entonces, de proponer perspectivas de revitalización lingüística ya no centradas en las denominadas lenguas modernas (cuyo enfoque, como ya se señaló, ha tenido como centro la escritura y las instituciones eurocéntricas), sino en resaltar la relevancia del aprendizaje de estas en un contexto de producción lingüística basado en la tradición y la comunicación oral desde la primacía de la transmisión intergeneracional como entorno sociocultural de aprendizaje. En este caso, su fundamento se encuentra en la comprensión de la importancia central de los agentes, los hablantes, quienes son los portadores del patrimonio lingüístico. Y esto se debe conectar indefectiblemente con perspectivas sociolingüísticas y etnolingüísticas, a fin de abordar la situación de minorización en que se encuentran las lenguas originarias.

En el contexto de lo señalado, abogamos por la promoción de una epistemología local/situada, referida en sus criterios de validación y operatividad a la observación y comunicación de una comunidad de hablantes, y expresada en un conjunto compartido de vivencias en los distintos ámbitos que conforman su mundo social y cultural, y cuya expresión, en este caso, es el mapunzugun. Así, se destaca "empoderamiento" de la comunidad de hablantes, entendido como la "participación y apropiación activas que hacen los hablantes de las iniciativas y estrategias que favorecen la posibilidad de fortalecer las lenguas y culturas amenazadas" $^{24}$. Es la generación y aplicación activa de los conocimientos que una comunidad se otorga, y opera como política de vitalización cultural y social a partir del uso de su lengua en un ámbito vivencial determinado. Asume que es la comunidad de hablantes la que desarrolla desde lo cotidiano el mundo lingüístico en el que habita y es este mundo activo la esencia de la particularización del conocimiento y su operacionalidad concreta, a fin de satisfacer sus necesidades de producción y reproducción cultural. Por lo tanto, el relevamiento de metodologías de enseñanza del mapunzugun debería situarse dentro de una coherencia epistemológica, lo que favorecería su legitimación dentro de la comunidad de hablantes, así como su articulación validada localmente con el PEIB.

Es en este sentido que la presente propuesta se sitúa, en un sentido amplio, dentro de una lingüística aplicada, ya que propone soluciones concretas relativas a la comunicación de y desde la realidad lingüística del mapunzugun en su contexto social, político y territorial de uso, a partir de "la necesidad de intervenir en las lenguas - sea en su código, sea en su estatus como modo de superar los posibles desequilibrios existentes" ${ }^{25}$. En un sentido más específico, pretende desarrollar políticas de revitalización lingüística de una lengua minorizada (en este caso, el mapunzugun), cuyo eje sea la expresión de metodologías de

24 José Antonio Flores Farfán, "El potencial de las artes y los medios audiovisuales en la revitalización lingüística", Revista de Lingüística Teórica y Aplicada, n.o 51, fasc. 1 (2013): 38.

25 Susana Pastor Cesteros, Aprendizaje de segundas lenguas. Lingüística aplicada a la enseñanza de idiomas (Alicante: Universidad de Alicante, 2006). 
enseñanza y aprendizaje que contengan y posibiliten la emergencia de su propia visión de mundo lingüístico. Así, la enseñanza estará centrada en la epistemología de la lengua y es a partir de esta que se desarrollará la construcción del proceso de revitalización.

En resumen, se trata de generar y promover procesos relacionados con la adquisición, el aprendizaje y la enseñanza de la lengua, a través del desarrollo de propuestas que incluyen enfoques y métodos cuyo centro sea la propia epistemología de lengua y sus implicancias prácticas en los niveles psicológicos y sociocognitivos que los hablantes consideren pertinentes.

\section{Descripción sociohistórica del mapunzugun}

Un aspecto relevante dentro de la concepción del mapunzugun como sistema lingüístico, y que cualquier iniciativa de revitalización debe tener presente, está relacionado con la descripción general de la lengua en términos sociohistóricos, tanto desde los cronistas y misioneros, como por parte de los primeros investigadores. En efecto, estos trabajos describen los rasgos principales del mapunzugun, en términos históricos, culturales y lingüísticos, partiendo por su presencia en términos territoriales. Así, estas referencias nos muestran, por ejemplo, las áreas que comprendía el uso del mapunzugun en tiempos de la conquista española: entre Coquimbo y las Guaitecas, de norte a sur, y hasta el océano Atlántico, por el este ${ }^{26}$. Como señala Valdivia ${ }^{27}$ :

[...] en todo el reino de Chile no ay mas de esta lengua que corre desde la Ciudad de Coquimbo y sus términos, hasta las yslas de Chilue y mas adelante por espacio casi de quatrocientas leguas de Norte a Sur que es la longitud del Reyno de Chile [...] aunque en diversas provincias de estos indios ay algunos vocablos diferentes, pero no son todos los nombres verbos y adverbios, y así los preceptos y reglas desta Arte son generales para todas las provincias.

Otro aspecto importante que se ha relevado históricamente se refiere a las escasas diferencias lingüísticas encontradas a lo largo del waj mapu, especialmente en el plano léxico y gramatical, y la dificultad de trazar alguna conexión sólida con otras lenguas del continente. Respecto de esto último, Molina señala que la lengua chilena es "única" en relación con las lenguas que se hablan en América, especialmente en cuanto a su estructura ${ }^{28}$. Esto es tratado también en la actualidad por Moulian y Catrileo, en términos de comparación entre el mapunzugun y otras lenguas ${ }^{29}$. En efecto, si bien el mapudungun se relaciona en términos de

26 Luis de Valdivia, Arte, y gramática general de la lengua que corre en todo el Reyno de Chile (Lima: 1606. Versión digital de la Biblioteca de la Universidad de Valladolid); Esteban Erize, Diccionario comentado mapuche-español. Araucano, pehuenche pampa, picunche rancülche huilliche (Bahía Blanca: Yepun, 1960); Ernesto Wilhelm de Moesbach, Idioma Mapuche (Villarrica: Imprenta Padre Las Casas, 1962).

27 Valdivia, Arte y gramática general, s. p.

28 Juan Ignacio Molina, Compendio de la Historia Civil General del Reyno de Chile (Madrid: Imprenta de Antonio de Sancha, 1795).

29 Rodrigo Moulian Tesmer y María Catrileo Chiguailaf, "Kamaska, kamarikun and müchulla: Loaned words and crossroads of mea- 
préstamos lingüísticos con otras lenguas de América, mantiene, sin embargo, su carácter de lengua "aislada" en cuanto a su adscripción a familias lingüísticas del continente; esto a pesar de los intentos por buscar conexiones consistentes.

\section{Regularidad y precisión de la lengua}

La unidad del mapunzugun y su regularidad es descrita y destacada, entre otros, por Valdivia ${ }^{30}$ y por Molina. Este último autor, por ejemplo, plantea que

[...] todo en ella es reglado, por decirlo así, con un mecanismo geométrico, donde se distingue un gran artificio con una suma simplicidad, y una relación tan ordenada y constante entre los preceptos gramaticales, que dependiendo siempre los subsiguientes de los antecedentes, su retórica se hace fácil, y se puede aprender cómodamente en pocos días. ${ }^{31}$

A partir de las afirmaciones del autor, es posible sostener que el mapunzugun constituye un sistema de comunicación normado lingüística y culturalmente; ello supone, a su vez, que el conocer y sistematizar el orden señalado facilita el aprendizaje de la lengua ${ }^{32}$.

Lo registrado por Molina también es manifestado por Moesbach, quien señala:

Se distingue por un vocabulario abundante, gran regularidad de todas las operaciones gramaticales; un mecanismo verbal muy detallado que facilita la expresión límpida de las modificaciones del pensamiento; sintaxis sencilla con frases casi siempre coordinadas y una fonética de condiciones estable y sonora; por medio de numerosos afijos y partículas intercaladas consigue una precisión y concisión casi inimitables. $^{33}$

Augusta plantea que es una lengua que destaca por su "sencilla y lógica estructura, la riqueza de sus formas verbales, la precisión y claridad de dicción y la facilidad con que da expresión a todo modo de pensar y sentir" 34 . Havestadt, por su parte, señala:

[...] la lengua de los araucanos aunque lo es de bárbaros, no solamente no es bárbara, sino que aventaja a las demás lenguas, así como los Andes sobresalen entre las demás montañas; de manera que a quien la posea le parecerá ver las demás como de lejos y bajo sus pies, conociendo claramente cuánto derecho puede reconvenir a

ning in the central and southern andean space", Alpha, n.o 37 (2013): 249-263.

30 Valdivia, Arte y gramática general.

31 Molina, Compendio de la Historia, 6.

32 Aquí cabe, entonces, la pregunta: ¿por qué el aprendizaje de la lengua en la actualidad presenta tantas dificultades, especialmente para las nuevas generaciones y más aún en contextos formales como el educativo? Indudablemente, es porque influyen otros factores asociados al aprendizaje con las actitudes lingüísticas, no solo de quienes aprenden la lengua, sino también de quienes la enseñan y promueven.

33 Moesbach, Idioma Mapuche.

34 Fray Félix José de Augusta, Lecturas Araucanas (Valdivia, Chile: Imprenta de la Prefectura Apostólica, 1910), 1. 
Aprendizaje del mapunzugun desde metodologías propias:

perspectivas y avances para la revitalización de lenguas originarias

cualquiera que no sea araucano: si el idioma tuyo es bueno el de los araucanos tiene la preferencia. ${ }^{35}$

Más adelante, y con respecto al nivel léxico, Molina caracteriza la lengua mapunche en los siguientes términos:

La lengua de Chile es de tal modo copiosa, que á juicio de todos aquellos que la han poseído con alguna perfección, se necesitaría mas de un grueso volumen para hacer de ella un completo diccionario, pues que a mas de la voces radicales, que son muchísimas, el uso de las composiciones es allí tan freqüente, que en cierta manera puede decirse que en esto consista la esencia de aquella lengua. Cada verbo, ó por derivación ó por unión, se hace raiz de otros inumerables verbos y nombres, así adjetivos como substantivos, los quales reproducen otros secundarios, modificándose en cien maneras diferentes. ${ }^{36}$

Y, en seguida, señala que:

No hay en ella parte alguna de la oración de la qual no pueda formarse un verbo peculiar con solo añadirle en el fin una $\boldsymbol{n}$. También de las partículas más simple derivan varios verbos propios, que comunican una gran precisión y fuerza al discurso. Pero lo que verdaderamente sorprende en esta lengua es que no se encuentra en ella nombre alguno ni verbo anómalo. ${ }^{37}$

Estos rasgos del mapunzugun han sido destacados también en nuestros días por diversos autores. Por ejemplo, Bengoa expresa que es admirable la capacidad descriptiva de las voces y la facilidad acumulativa, las que constituyen un carácter prominente de la lengua ${ }^{38}$. Esta se evidencia en el conocimiento acerca del mundo natural para señalar características detalladas de cerros, espacios, plantas, animales, aves, situaciones socioculturales, etcétera.

\section{Capacidad de abstraer de la lengua}

Este aspecto no siempre ha sido reconocido plenamente por quienes han abordado el estudio del mapunzugun, principalmente porque ha primado la relación hegemónica, donde la base de comparación ha sido la lengua colonial, lo cual expresa la distancia que ha existido en los estudios para comprender las bases lingüísticas y culturales mapunche; sin embargo, Augusta expresa una visión distinta, cuando sostiene que esta lengua permite expresar "todo el modo

35 Bernardi Havestadt, Chilidúgu sive res chilenses vel Descriptio Status tum naturalis, tum civilis, cum moralis Regni populique Chilensis, inserta suis locis perfectæ ad Chilensem Limguam Manuductioni Deo O.M. multis ac miris modis Juvante (Lipsiae: Typis B. G. Teubneri, 1777).

36 Molina, Compendio de la Historia; María Palumbo. Educación en Movimientos Populares Rurales: Un Estado del Arte. Revista Historia de la Educación Latinoamericana Vol. 18 no.26 (2016): 219-40. https://doi.org/10.19053/01227238.4373.

37 Ibíd.

38 José Bengoa Cabello, Historia del pueblo mapuche (Santiago de Chile: Editorial LOM, 1987). 
de pensar y sentir"; lo cual, en el caso del mapunzugun, se vincula directamente con aspectos de orden filosófico, como es el relevamiento del az mapu como ordenamiento del territorio bajo la idea de derechos y pertenencia social, identitaria y política.

Lo anterior implica, entonces, la comprensión de diversos rasgos del mapunzugun, los que solamente pueden ser aprehendidos desde el propio marco lingüístico y cultural mapunche. En efecto, cualquier búsqueda de sentido desde el español no cumplirá plenamente con los requisitos de producción comprensivo/ comunicativa que el mapunzugun contiene, lo que también se da en sentido inverso. Diversos trabajos han ido evidenciando esta capacidad del mapunzugun $^{39}$, lo que da cuenta de su particularidad como expresión y acción lingüística de realidad.

\section{Mapuzungun en el marco de la Educación Intercultural Bilingüe}

A partir de la década del setenta del siglo pasado, las lenguas originarias se visualizan como parte de la preocupación de estudios en el ámbito de las ciencias sociales y la educación, debido en gran parte a la visibilización de movimientos sociales indígenas, los cuales promueven la reivindicación de derechos ancestrales de diverso orden; entre ellos, aquellos concernientes al patrimonio lingüístico y cultural. Estos estudios surgen fundamentalmente como respuesta al modelo educativo colonial, que desarrolla procesos de enajenación cultural y lingüística; en particular, la prohibición factual del uso de la lengua por parte de las nuevas generaciones, situación observada aún en la actualidad ${ }^{40}$. Como respuesta a esta problemática, surge en Chile la Educación Intercultural Bilingüe, la cual pretende, por un lado, responder a los procesos de enajenación cultural y lingüística ejercidos desde el español sobre el mapunzugun, así como buscar pertinencia al currículum educativo en contextos indígenas, a fin de abordar, entre otros aspectos, la identidad y pertinencia de prácticas educativas que permitan y favorezcan la expresión y continuidad de la lengua originaria, dentro del contexto de mejora de la calidad de la educación de la población indígena; sin embargo, como hemos señalado en páginas precedentes, la aplicación del PEIB no ha logrado responder a los lineamientos estratégicos que se ha propuesto. Una de las razones es que la EIB se orienta y dirige a las poblaciones indígenas desde una perspectiva curricular propia del modelo dominante, no considerando la incorporación de los modelos de enseñanza de la lengua contenidos en la expresión sociocultural de esta. En este contexto, la afirmación de Sichra, en cuanto a que la didáctica de la lengua originaria se ve obstaculizada debido a la ausencia de métodos apropiados y maestros formados con la especificidad

39 Desiderio Catriquir Colipan y Teresa Durán Pérez, "Kimeltuwün zugu: Modelo educativo mapunche”, en Patrimonio cultural mapunche. Derechos sociales y patrimonio institucional mapunche, comp. por Teresa Durán Pérez, Desiderio Catriquir Colipan \& Arturo Hernández Sallés, vol. III (Temuco: Ediciones de Universidad Católica de Temuco, 2007).

40 Teresa Durán Pérez y Nelly Ramos Pizarro, "Incorporación del español por los mapuches del centro sur de Chile durante el siglo XIX”, Lenguas Modernas, n.o 14 (1987): 179-196. 
necesaria para la enseñanza de la lengua indígena ${ }^{41}$, referida a la enseñanza del quechua, puede ser extrapolada a la enseñanza del mapunzugun.

Enseñanza y aprendizaje del mapuzungun como segunda lengua desde las epistemologías y metodologías propias

En términos generales, se entiende por segunda lengua aquella que se "halla en el contexto inmediato del aprendiz, y este puede entrar en contacto con ella, escucharla o hablarla, usarla, en definitiva, también fuera del aula" ${ }^{42}$ y que implica, de acuerdo con Pastor, "el favorecer el aprendizaje de una lengua no nativa por parte de alumnos que no la dominan por completo, o incluso en absoluto, y el consiguiente desarrollo de la competencia comunicativa en esa nueva lengua; ello supone una reestructuración cognitiva, perceptual y relacional de las competencias ya adquiridas en la lengua materna" ${ }^{43}$. Este último aspecto de la enseñanza de segundas lenguas, la Competencia Comunicativa, resulta ser muy relevante al momento de proponer y aplicar políticas de revitalización lingüística, ya que permite centrar el aprendizaje de la lengua en sus supuestos constitutivos como expresión sociocultural de realidad. Es lo que desde la Etnolingüística Cognitiva Bartmiński denomina "visión de mundo lingüístico", la cual implica una interpretación de la realidad profundamente arraigada en el lenguaje y que puede ser expresada en la forma de juicios acerca del mundo, de las personas, cosas o eventos y que unifica a las personas en un entorno social dado, crea una comunidad de pensamiento, sentimientos y valores ${ }^{44}$.

La Competencia Comunicativa no implica solamente la mera descripción de los patrones de uso lingüístico, sino que debe permitir a los lingüistas preocupados por la comunicación en grupos humanos el concentrarse en aspectos del conocimiento compartido y de las habilidades cognitivas de la comunidad de hablantes, tal como señala Gumperz ${ }^{45}$. Por su parte, Weyers y Canale, desde una perspectiva más cercana a la Lingüística Aplicada, definen la Competencia Comunicativa como sistemas subyacentes de conocimientos y habilidades requeridas para la comunicación, y la dividen en cuatro subcompetencias asociadas a áreas particulares de conocimiento y habilidad: competencia gramatical (dominio del código lingüístico tanto verbal como no verbal), competencia sociolingüística (adecuación tanto de la forma como del significado del discurso al contexto en que se produce), competencia discursiva (conocimiento necesario para dar conexión a un texto tanto oral como escrito y en diferentes géneros) y competencia estratégica (relativa a aquellas técnicas e instrumentos, verbales y

41 Inge Sichra, Enseñanza de la lengua indígena e interculturalidad: ¿entre la realidad y el deseo? Investigación sobre la enseñanza del quechua en dos colegios particulares en Cochabamba (Cochabamba, Bolivia: PROEIB Andes, 2006), 8.

42 https://www.apuntes.com/magisterio/niveles-de-concrecion-de-los-objetivos

43 Pastor Cesteros, Aprendizaje de segundas lenguas, 66 y 68.

44 Jerzy Bartmiński, Aspects of Cognitive Ethnolinguistics (United Kingdom: MPG Books Group, 2013).

45 John Gumperz, "Las bases lingüísticas de la competencia comunicativa”, en Etnografía del habla. Textos fundacionales, ed. de Lucía Golluscio (Buenos Aires: Eudeba, 2002). 
no verbales, que permiten subsanar los problemas o interrupciones en la comunicación $)^{46}$. En el contexto del presente artículo, resulta relevante entender que la competencia comunicativa en todas sus dimensiones se encuentra supeditada a los alcances epistemológicos de la lengua. Es decir, la competencia comunicativa será entendida como la comprensión, comunicación e interpretación adecuada de la visión de mundo lingüístico que la lengua despliega y que permite su unidad, y a la vez su diferencia, como sistema.

En este sentido, la enseñanza de una lengua originaria se encuentra acompañada y entrelazada con la cultura y tradiciones de sus hablantes, por lo que debe ser entendida como un elemento relevante dentro de la construcción de una identidad indígena. Así, entonces, las tradiciones y cultura de la comunidad lingüística deberán ser adquiridas dentro de la enseñanza y aprendizaje de la lengua. En palabras de Fishman ${ }^{47}$, lenguas específicas están relacionadas con culturas específicas y acompañan a la identidad cultural en el nivel del hacer, en el nivel del conocer y en el nivel del ser. Para el caso que nos ocupa, el mapunzugun, al igual que el español de Chile, constituye la expresión comunicativa/ comprensiva de una visión lingüística de mundo cuyo uso es la emergencia de una identidad cultural.

Pese a esto, los procesos de revitalización de lenguas originarias se han desarrollado fundamentalmente a partir de su inclusión dentro de la educación formal, es decir, desde estrategias, métodos y recursos de la lengua dominante, antes que desde las estrategias que la lengua minorizada contiene como propuesta de enseñanza. Es por ello que un aspecto central dentro de la presente propuesta, y como ya se insinuó mediante la incorporación del concepto de epistemologías y metodologías propias, es promover la enseñanza del mapunzugun a partir de la transmisión intergeneracional a un nivel comunitario y familiar. Esto resulta particularmente pertinente si consideramos que los procesos de revitalización lingüística se han desarrollado a lo largo de la historia como esfuerzos de aprendizaje por parte de una comunidad o individuos cuando la transmisión regular en el hogar ha fallado ${ }^{48}$. De acuerdo con esto, el uso de la lengua en un contexto familiar y comunitario resulta esencial si se pretende revertir la pérdida de vitalidad de esta, relevando y ampliando, desde una perspectiva social propia, y en cierta medida íntima, la relación entre la visión sociocognitiva del mundo lingüístico mapunzugun y su despliegue creador de ámbitos de uso y comprensión de mundo.

Así, entonces, la presente propuesta de revitalización del mapunzugun tiene como uno de sus ejes centrales el desarrollo de procesos de transmisión $-\mathrm{y}$, por lo tanto, de metodologías de enseñanza de la lengua - que permitan la reproducción sociocultural de la visión de mundo lingüístico del mapunzugun. En

\footnotetext{
46 Joseph Weyers y Germán Canale, “Tuteo and voseo in the classroom: Linguistic attitudes among Montevidean educators", Spanish in Context, n.o 10 (2013): 371-389.

47 Joshua A. Fishman, ed., Can Threatened Languages Be Saved? Reversing Language Shift, Revisited: A 21st Century Perspective (Clevedon: Multilingual Matters, 2001).

48 Hinton y Hale, The Green Book.
} 
este contexto, la revitalización, que debe favorecer el aumento de contextos de uso y de la cantidad de hablantes, debe situar la lengua dentro de una práctica comunicativa intergeneracional cotidiana y centrarse en la epistemología de la lengua como eje de su transmisión comprensivo/comunicativa. A partir de este posicionamiento, se deben generar prácticas que permitan potenciar la aplicación en el aula del PEIB en dos sentidos: en primer lugar, acompañar y favorecer desde la comunidad las estrategias del programa y, en segundo lugar, aumentar el prestigio y estatus de la lengua, a fin de favorecer el desempeño dentro del aula del educador tradicional. De este modo, el centro del proceso de revitalización lingüística estará situado en contextos sociales y culturales propios de la comunidad de hablantes y desde estos se acompañará y potenciará la enseñanza formal del mapunzugun dentro de la escuela.

En resumen, se trata del desarrollo y aplicación de metodologías de enseñanza del mapunzugun como segunda lengua que provengan de los principios epistemológicos de construcción de la visión de mundo lingüístico del mapunzugun, y que, a su vez, potencien la participación institucional (escuela) dentro de su reconocimiento identitario. Esto, de acuerdo con nuestra perspectiva, favorecería el aumento de la comprensión y pertinencia de la enseñanza formal del mapuzungun en la escuela y del rol y estatus del educador tradicional como agente activo dentro del reconocimiento estatal de la existencia de la realidad cultural y lingüística mapunche. Así se rompería con la relación de subordinación del educador tradicional respecto del profesor (con educación universitaria formal), relación que influye de manera relevante en la percepción que sobre el estatus de la lengua tiene la comunidad educativa ${ }^{49}$.

Aproximación al mapun kimün - rakizuam en torno al zuam y zugu como perspectivas propias

De acuerdo con lo señalado, y lo que se expondrá en adelante, el presente artículo es el resultado de, por una parte, una revisión bibliográfica (documentación lingüística) en torno a la enseñanza-aprendizaje del mapunzugun $\mathrm{y}$, por otra, de una mirada reflexiva en torno al estado de esta lengua y sus posibilidades de aprendizaje/revitalización desde marcos lingüísticos y culturales propios. En este contexto, se relevan conceptos que provienen del mapunzugun en cuanto expresión del kimün que los miembros de diferentes lof mapu plantean en los güxam, referidos al estado y proyección de su lengua; en particular, a la preocupación por su pérdida, especialmente entre los jóvenes. En efecto, el presente trabajo expone nociones propias derivadas de interacciones indagativas ("trabajo de campo": propuesta y realización de güxam) o participación en distintos actos ceremoniales, recogidas y relevadas como hablante nativo y conocedor del mapunzugun por el autor principal del presente trabajo (Gabriel Llanquinao). De este modo, se genera una perspectiva reflexiva desde el mapun kimün y mapun

49 Lagos, "El Programa de Educación", 84-94. 
rakizuam, proyectada académicamente como marco "teórico" y "metodológico" propio y que se constituye en una aproximación discursiva que se orienta desde y hacia el mapunzugun.

Así, desde un punto de vista teórico y metodológico, este ejercicio reflexivo intenta superar las descripciones señaladas en la documentación revisada, de tal modo que los conceptos que emergen desde el mapunzugun puedan desplegarse desde sus propios rasgos y denotaciones, generando así una cadena de conceptos interrelacionados que se presentan como alternativas socioculturales propias de comprensión, aprendizaje y revitalización de la lengua. En este contexto, y en un sentido académico, dicho ejercicio reflexivo se puede situar fundamentalmente dentro del paradigma indígena de investigación, "el cual reside en la ciencia nativa, que es lo vivencial, la experiencia, el saber acumulado y compartido" 50 .

Como señalábamos, el mapun kimün y el mapun rakizuam ocupan un lugar central en esta presentación. En este contexto, valga señalar que el concepto de kimün tiene su base en la noción de kim, entendido como conocimiento, saber, consciencia, lucidez; kimün, entonces, se refiere a lo conocido, sabido o aprehendido, como también "saber, entender, conocer" ${ }^{\prime \prime 1}$. En diversas expresiones usadas por hablantes del mapunzugun, kimün se usa como el conocimiento construido respecto de un objeto de conocimiento que es el zugu, el que será expresado verbalmente bajo la idea de zugun, esto es, como un relato. Por lo tanto, siempre en la base de un zugu habrá un kimün y sobre el kimün habrá un sustento que es la racionalidad o pensamiento, es decir, un rakizuam, en este caso mapunche.

El rakizuam reconoce como centro o idea principal el zuam, esto es, aquello inherente a lo humano, una necesidad y sentido de estar en el mundo. Todos tenemos un zuam, que surge desde necesidades elementales relacionadas con un estar en el tiempo y en un espacio en el cual existir, vivir el mundo y en el mundo, aquello que le da sentido a la vida, en un contexto, situación o visión de mundo particular, en este caso mapunche. Así, respecto del zuam se dice che ta niekey ta zuam ('las personas suelen tener zuam'). Por su parte, raki o rakin es contar, enumerar, priorizar en términos de la importancia que tienen los distintos zuam, de manera que el raki moldea los zuam, configurando así el rakizuam, entendido como una totalidad, un modo de comprender y situar diversos objetos y situaciones del mundo, susceptible de ser concebido y abstraído en una manera de ejecutarse o realizarse. Así también, rakizuam es la reflexión sobre algo y es expresado en la idea de rakizuamün, esto es, pensar, reflexionar, meditar; en palabras de Erize, es "pensamiento, opinión" 52 .

En consecuencia, metodológicamente, todo el quehacer u objeto de conocimiento desde lo mapunche - en este caso vinculado con el aprendizaje por parte

\footnotetext{
50 Weimar Iño Daza, "Epistemología pluralista, investigación y descolonización. Aproximaciones al paradigma indígena”, RevIISE, n.o 9 (2017): 111-125.

51 Erize, Diccionario comentado.

52 Ibíd.
} 
de las nuevas generaciones - supone una aproximación y visibilización desde lo propio. Así, se deberán reconocer necesariamente como marcos propios el kimün y el rakizuam (en el modelo de conocimiento y pensamiento mapunche, se dirá mapun kimün y mapun rakizuam). Esta aproximación es válida y necesaria, toda vez que permite situar el proceso de indagación desde referentes propios, y se constituye, como señalan los hablantes nativos, en wüxampüram kimün, esto es, en un modo de 'levantar el conocimiento propio'.

Otras palabras plasman también distintos recursos comunicativos que permiten (re)construir, adentrarse y continuar el despliegue de ámbitos de conocimiento mapunche, en la medida en que se ubican en un mapun kimün y en un mapun rakizuam. Uno de estos recursos es el güxam, que se despliega como diálogo, conversación, retórica. El orientarse en el marco de un güxam, permite situar temas y asuntos que conciernen a la vida cotidiana de las personas, en el contexto de la familia y de la comunidad. Es importante enfatizar que en un proceso de indagación desde el modelo mapunche, se debe contar con un güxam, es decir, se debe llevar un güxam a la gente para compartir y desde allí averiguar y luego profundizar. En el contexto de nuestro trabajo, esto es fundamental, pues se relaciona con el aprendizaje del mapunzugun por parte de las nuevas generaciones; dicho en mapunzugun: ñi kimken kewüh ta wekeche (aprendizaje de la lengua en las nuevas generaciones). En efecto, desplegar un güxam supone contar con algunas aproximaciones desde las versiones que la gente tiene y relacionarlas con el conocimiento construido en la familia y la comunidad (lof).

Por otro lado, en el contexto de nuestra participación en ceremoniales y de observación en situaciones de comunicación en la vida cotidiana, encontramos con los hablantes nativos diversas expresiones atingentes a la pérdida de la lengua. En efecto, descubrimos ideas o nociones que orientan su proceso, como, por ejemplo, cuando al advertir dicha pérdida, especialmente en las nuevas generaciones, se dice mapunzuguwelay ta pu wekeche (los jóvenes ya no hablan mapunzugun) y por otro lado, algunas expresiones que dan cuenta de la posibilidad de su recuperación.

En este contexto, a lo menos tres conceptos se interrelacionan, a saber: wiñokintugetual, como posibilidad de reconstrucción desde nociones propias en relación con el pasado; günaytugetual, entendido como revaloración y aprecio hacia lo propio, que consiste en recuperar la lengua desde las matrices lingüísticas y culturales propias; y poyenyegetual, reconstruir la lengua desde la valoración propia, incorporando afectos en cuanto patrimonio compartido por la comunidad.

Todo lo anterior nos indica que los miembros mapunche hablantes del mapunzugun no solo advierten y muestran la pérdida progresiva de la lengua, sino que también plantean nociones y alternativas propias de revitalización.

Otros conceptos refieren a la reflexión en torno a situaciones o temáticas que tienen implicancias prácticas. Uno de ellos es güneltun y de manera práctica güneltugeal zugu, en referencia a un modo de darse cuenta, reflexionar en torno a una temática sensible, distinguir niveles de situaciones que permiten vislumbrar alternativas de acción, así como un modo de comprender y conocer; aquí günel- 
tun está relacionado, en el ámbito de la lengua, con procesos metalingüísticos. Otro concepto es zugunyegeal, el cual conlleva un nivel de práctica permanente y sostenido; dicho de otro modo, llevar a través de un zugu y expresarlo en un zugun permanente. Finalmente, surge también zapigetuael, cuyo concepto base es el zapin, que implica la interpelación desde el modelo educativo y formativo mapunche al cultivo y desarrollo de, en este caso, la lengua; esta idea de cultivo tiene relación con el cuidado y depurado de la lengua y los conocimientos concernientes a ella. Aquí, la terminación ael, en el sistema de afijación de conceptos en mapunzugun, hace referencia a la posibilidad de futuro, aquello que puede ser comprendido por otros, y por tanto generalizable, e implica también niveles de consenso o acuerdos.

Un tercer nivel de conceptos contiene ideas referidas a la reconstrucción, proyección y movilización respecto de iniciativas vinculadas al ámbito de la revitalización desde las nociones propias. Así, tenemos la noción de amulgetuael, como aquello que puede ser conducido y orientado, pero que previamente debe ser sistematizado y ordenado; en su sentido literal, quiere decir "aquello que puede volver a ser proyectado". Wenuntugetuael, por su parte, implica que se requiere una manera de situar y resituar la lengua, es decir, como construcción y reconstrucción de los conocimientos, lo cual se condice con la advertencia de los hablantes en cuanto al empobrecimiento en el uso del mapunzugun en la actualidad que cruza las distintas generaciones (se dice que ya no siempre hay kimün en el uso del mapunzugun). Por último, miyawülgetual tiene distintas connotaciones; entre ellas, superar la visión negativa acerca del uso de la lengua en distintos espacios, particularmente, en contextos de relaciones interétnicas e interculturales. Del mismo modo, significa una reapropiación de la lengua como patrimonio cultural y, en consecuencia, vinculado a la identidad mapunche. Finalmente, una tercera idea asociada con este concepto, señala que la lengua debe ser portada y usada por los hablantes.

\section{CONCLUSIÓN}

El trabajo presentado permite vislumbrar alternativas para el aprendizaje de lenguas originarias (específicamente, respecto del mapunzugun o lengua mapunche), considerando los marcos teóricos y metodológicos apropiados, los que, para efectos de la presente propuesta, deben centrarse en y desde la valoración del mapun kimün y mapun rakizuam como eje de la mantención y revitalización del mapunzugun: lo importante no es tan solo la lengua, sino la vida y forma de vivir que esta contiene.

Por otra parte, este trabajo permitió generar y desplegar una perspectiva diferente, complementaria y actualizada acerca de las nociones propias contenidas en la documentación lingüística descrita, las que se unen con nociones propias sobre aspectos metodológicos planteados por los hablantes de la lengua tanto en contextos familiares como en el ámbito de los ceremoniales. Es importante seña- 
lar que los diversos estudios señalados en el presente artículo proveen información y descripción que facilitan perspectivas de actualización de metodologías de estudios, así como acercamientos con hablantes nativos del mapunzugun, a fin de generar unidades temáticas para profundizar y actualizar los conocimientos, en especial lo vinculado al ámbito del conocimiento lingüístico. En este sentido, la Educación Intercultural Bilingüe emerge como un campo propicio para generar dichos acercamientos, pero ahora con base en las experiencias desarrolladas en el ámbito de la enseñanza del patrimonio lingüístico de las comunidades, así como su relevancia en el campo del bilingüismo, toda vez que en América Latina en general surge como vertiente que visibiliza las lenguas originarias más allá de los estudios descriptivos.

De este modo, entonces, esta presentación enfatiza la necesidad de desarrollar y aplicar metodologías de enseñanza que provengan de la práctica sociocultural de la lengua a revitalizar, situando en un segundo plano las lógicas teórico/pedagógicas provenientes de la lengua dominante. En este contexto, situar dicho proceso en el marco del mapun kimün y el mapun rakizuam, tal como hemos planteado y justificado, se constituye en un paso sustantivo que debe desplegarse con sus distintos alcances e implicancias.

\section{FUENTES}

Apuntes para todos los estudiantes y cursos. https://www.apuntes.com/

Ministerio de Educación. https://www.mineduc.cl/.

\section{REFERENCIAS}

Augusta, fray Félix José de. Lecturas Araucanas. Valdivia, Chile: Imprenta de la Prefectura Apostólica, 1910.

Bartmiński, Jerzy. Aspects of Cognitive Ethnolinguistics. United Kingdom: MPG Books Group, 2013.

Becerra, Rodrigo. “Una propuesta funcional y tipológica de descriptores sintácticos para el mapudungun". Alpha, n. 32 (2011): 111-125.

Becerra, Rodrigo. “Expresión de la causalidad en la lengua mapuche". Tesis de maestría en Lingüística Aplicada, Universidad de Concepción, Chile, 2009.

Bengoa, José. Historia del pueblo mapuche. Santiago de Chile: Editorial LOM, 1987.

Catrileo Chiguailaf, María. La lengua mapuche en el siglo XXI. Valdivia: Universal Austral de Chile, 2010.

Catrileo Chiguailaf, María. "Revitalización de la lengua mapuche en Chile". Documentos Lingüísticos y Literarios, n. 28 (2005): 10-17.

Catriquir Colipan, Desiderio y Teresa Durán Pérez. “Kimeltuwün zugu: Modelo educativo mapunche". En Patrimonio cultural mapunche. Derechos sociales y patrimonio institucional mapunche. Compilado por Teresa Durán, Desiderio Catriquir \& Arturo Hernández. Volumen III. Temuco: Ediciones de Universidad Católica de Temuco, 2007.

Corporación Nacional de Desarrollo Indígena (CONADI) (1993), Ley indígena n.ํㅜ 19.253. D. of. 5-10-1993. Santiago.

Darquennes, Jeroen. "Paths to Language Revitalization". En Contact Linguistics and Language Minorities. Ed. de Jeroen Darquennes. Vol. XXX de Plurilingua. St. Augustin: Asgard, 2007. 
Durán Pérez, Teresa y Nelly Ramos Pizarro. “Incorporación del español por los mapuches del centro sur de Chile durante el siglo XIX". Lenguas Modernas, n. 14 (1987): 179-196.

Erize, Esteban. Diccionario comentado mapuche-español. Araucano, pehuenche pampa, picunche rancülche huilliche. Bahía Blanca: Yepun, 1960.

Fishman, Joshua A., ed. Can Threatened Languages Be Saved? Reversing Language Shift, Revisited: A 21st Century Perspective. Clevedon: Multilingual Matters, 2001.

Fishman, Joshua A. Reversing Language Shift: Theoretical and Empirical Foundations of Assistance to Threatened Languages. Clevedon: Multilingual Matters, 1991.

Flores Farfán, José Antonio. “El potencial de las artes y los medios audiovisuales en la revitalización lingüística". Revista de Lingüística Teórica y Aplicada, n. ${ }^{\circ}$ 51, fasc. 1 (2013): 33-52.

Gumperz, John. "Las bases lingüísticas de la competencia comunicativa". En Etnografía del habla. Textos fundacionales. Ed. de Lucía Golluscio. Buenos Aires: Eudeba, 2002.

Gundermann Kröll, Hans, Jaqueline Canihuan Canihuan, Ernesto Castillo Narbona y Alejandro Clavería Cruz. Perfil sociolingüístico de comunidades mapuche de la VIII, IX y X Región. Informe de Resultados 2008. Santiago de Chile: Universidad Tecnológica Metropolitana-Conadi, 2008.

Gundermann Kröll, Hans, Jaqueline Canihuan Canihuan, Alejandro Clavería Cruz y César Faúndez Lagos. "Permanencia y desplazamiento, hipótesis acerca de la vitalidad del mapuzungun". Revista de Lingüística Teórica y Aplicada, n. ${ }^{\circ}$ 47, fasc. 1 (2009): 37-60.

Havestadt, Bernardi. Chilidúgu, sive res chilenses vel Descriptio Status tum naturalis, tum civilis, cum moralis Regni populique Chilensis inserta suis locis perfectæ ad Chilensem Limguam Manuductioni Deo O.M. multis ac miris modis Juvante. Lipsiae: Typis B. G. Teubneri, 1777.

Hernández, Arturo, Nelly Ramos y Rosa Huenchulaf. Gramática Básica de la Lengua Mapuche. Temuco: Universidad Católica de Temuco-Conadi, 2006.

Hinton, Leanne y Ken Hale, eds. The Green Book of Language Revitalization in Practice. San Diego, CA: Academic Press, 2001.

Iño, Weimar. "Epistemología pluralista, investigación y descolonización. Aproximaciones al paradigma indígena". RevIISE 9, n. ${ }^{\circ} 9$ (2017): 111-125.

King, Kendall. Language Revitalization Processes and Prospects. Clevedon: Multilingual Matters, 2001.

Lagos, Cristián. “El Programa de Educación Intercultural Bilingüe y sus resultados: ¿perpetuando la discriminación?". Pensamiento Educativo. Revista de Investigación Educacional Latinoamericana 52, n. ${ }^{\circ} 1$ (2015): 84-94.

Lagos, Cristián y Marco Espinoza. “La planificación lingüística de la lengua mapuche en Chile a través de la historia". Lenguas Modernas, n. ${ }^{\circ} 42$ (2013): 47-66.

Lagos, Cristián. “El mapudungún en Santiago de Chile: Vitalidad y representaciones sociales en los mapuches urbanos". Revista de Lingüística Teórica y Aplicada, n. ${ }^{\circ}$ 50, fasc. 1 (2012): 161-190.

Mariano, Héctor, Daniela Molina, Cristián Oyarzo, Cristián Lagos y Felipe Hasler. “¿Mongeley kam mongelelay chi mapudungun waria mew? Vitalidad y representación social del mapudungún en Santiago de Chile". Romanitas 4, n. ${ }^{\circ} 2$ (2010): 42-69.

Moesbach, Ernesto Wilhelm de. Idioma Mapuche. Villarrica: Imprenta Padre Las Casas, 1962.

Molina, Juan Ignacio. Compendio de la Historia Civil General del Reyno de Chile. Madrid: Imprenta de Antonio de Sancha, 1795.

Moulian Tesmer, Rodrigo y María Catrileo Chiguailaf. "Kamaska, kamarikun and müchulla: Loaned words and crossroads of meaning in the central and southern andean space". Alpha, n. ${ }^{\circ} 37$ (2013): 249-263.

Olate Vinet, Aldo y Marisol Henríquez Barahona. "Actitudes lingüísticas de profesores mapuche de Educación Básica: vigencia y enseñanza del mapudungun en el contexto educativo". Literatura y Lingüística, n. 22 (2010): 103-116.

Palumbo, María. Educación en Movimientos Populares Rurales: Un Estado del Arte. Revista Historia de la Educación Latinoamericana Vol. 18 no. 26 (2016): 219-40. https://doi. org/10.19053/01227238.4373.

Pastor Cesteros, Susana. Aprendizaje de segundas lenguas. Lingüística aplicada a la enseñanza de idiomas. Alicante: Universidad de Alicante, 2006.

Sadowsky, Scott, Juan Héctor Painequeo, Gastón Salamanca y Heriberto Avelino. "Mapudungun". Journal of the International Phonetic Association, n. ${ }^{\circ} 43$ (2013): 87-96. 
Salamanca, Gastón y Elizabeth Quintrileo. “El mapuche hablado en Tirúa: fonemas segmentales, fonotaxis y comparación con otras variedades". Revista de Lingüística Teórica y Aplicada, n. ${ }^{\circ}$ 47 (2009): 13-35.

Salas, Adalberto. El mapuche o araucano. Fonología, gramática y antología de cuentos. Santiago: Centro de Estudios Públicos, 2006.

Sánchez, Makarena y Gastón Salamanca. "El mapuche hablado en Lonquimay: Fonemas segmentales, fonotaxis y comparación con otras variedades". Literatura y Lingüística, n. 31 (2015): 295-332.

Sichra, Inge. Enseñanza de la lengua indígena e interculturalidad: ientre la realidad y el deseo? Investigación sobre la enseñanza del Quechua en dos colegios particulares en Cochabamba. Cochabamba, Bolivia: PROEIB Andes, 2006.

Strubell, Miquel. "Can Sociolinguistic Change be Planned?". En Proceedings of the 1st European Conference, "Private Foreign Language Education in Europe: Its contribution to the Multilingual and Multicultural Aspect of the European Union", 23-31. Thessaloniki: Palso, 1998.

Teillier Coronado, Fernando. “Configuración de realidad y lengua: bases de una epistemología del mapunzugun". Tesis de doctorado en Lingüística. Universidad de Concepción, Chile, 2015.

Teillier Coronado, Fernando. "Vitalidad lingüística del mapudungun en Chile y epistemología del hablante". Revista de Lingüística Teórica y Aplicada, n. ${ }^{\circ}$ 51, fasc. 1 (2013): 53-70.

Valdivia, Luis. Arte y gramática general de la Lengua que corre en todo el Reyno de Chile. Lima, 1606. Versión digital de la Biblioteca de la Universidad de Valladolid.

Weyers, Joseph R. y Germán Canale. "Tuteo and voseo in the classroom: Linguistic attitudes among Montevidean educators". Spanish in Context, n. ${ }^{\circ} 10$ (2013): 371-389.

Wittig, Fernando. 2009. “Desplazamiento y vigencia del mapudungún en Chile: un análisis desde el discurso reflexivo de los hablantes urbanos". Revista de Lingüística Teórica y Aplicada, n. ${ }^{\circ}$ 47, fasc. 2 (2009): 135-155.

Zúñiga, Fernando. “Mapudunguwelaymi am? ‘Acaso ya no hablas mapudungun?' Acerca del estado actual de la lengua mapuche". Estudios Públicos, n. ${ }^{\circ} 105$ (2007): 9-24.

Zúñiga, Fernando. Mapudungun. El habla mapuche. Santiago de Chile: Centro de Estudios Públicos, 2006.

\section{Cómo citar:}

Llanquinao Llanquinao, Gabriel; Salamanca Gutiérrez, Gastón; Teillier Coronado, Fernando. "Aprendizaje del mapunzugun desde metodologías propias: perspectivas y avances para la revitalización de lenguas originarias". Revista Historia de la Educación Latinoamericana. Vol. 21 No. 33 (2019): 121-143

https:https://doi.org/10.19053/01227238.9097

(c) (1) @ (9) Esta obra está bajo una licencia Creative Commons. Reconocimiento-No Comercial-Sin Obra Derivada 2.5 Colombia. 